\title{
Towards Simple and Robust Automation of Sustainable Supply Chain Communication
}

\author{
Gregor Grambow, Nicolas Mundbrod, Jens Kolb and Manfred Reichert \\ Institute of Databases and Information Systems \\ Ulm University, Germany \\ \{gregor.grambow, nicolas.mundbrod, jens.kolb, manfred.reichert\}@uni-ulm.de \\ http://www.uni-ulm.de/dbis
}

\begin{abstract}
In supply chains, companies are forced to produce in a more sustainable way. Amongst others, this involves the reporting of various sustainability indicators to legal entities. To gather necessary data from their suppliers, companies must employ long-running, crossorganizational data collection processes. Being dependent on many companies and contextual factors such processes imply great variability, are manually managed, and tend to be tedious and error prone. This paper proposes an approach for automated contextual process configuration that also supports humans with lightweight, correct modeling and execution of configurable processes.
\end{abstract}

Key words: Process Configuration, Business Process Variability, Data Collection, Sustainability, Supply Chain

\section{Introduction}

In many domains of modern industry, sustainability has become increasingly important. Companies are forced by legal regulations and customer demands to a more sustainable production. This involves the reporting of various sustainability indicators, such as the amount of lead in a certain product. However, in a supply chain, this becomes a challenge as most products are the result of the collaboration of multiple companies. Consequently, reporting companies must employ long-running, cross-organizational data collection processes to obtain required indicators from their suppliers as well. Furthermore, as sustainability is an emerging topic, most companies do not have a standardized approach to such reporting. Thus, the latter still is tedious and error prone.

In the SustainHuh ${ }^{1}$ project, we have identified a set of core challenges for such data collection processes by investigating use cases from companies in the electronics and automotive domain. The first challenge concerns the selection of the parties involved. As reporting is manually managed, it is often not clear, which suppliers or service providers need to be involved for retrieving a certain indicator. Furthermore, as different companies employ different approaches, data

\footnotetext{
${ }^{1}$ SustainHub (Project No.283130) is a collaborative project within the 7th Framework Programme of the European Commission (Topic ENV.2011.3.1.9-1, Eco-innovation).
} 
formats, and reporting tools, getting access to the data is often problematic. Being dependent on a myriad of contextual influences, each data collection process is almost unique. Thus, usually, many variants of the same request exist usually that cannot be reused. Moreover, the meta data, on which these variants depend, is mostly managed implicitly and thus obscure (see [1] for more information on these challenges).

\section{A Process-based Approach for Data Collection}

In the SustainHub project, we are developing an approach to support such data collection in a semi-automated fashion. As basis for this approach, we realize the data collection processes through explicitly modeled processes enacted in a PAIS (Process-Aware Information System). Thus, a set of issues can already be dealt with as the processes are now explicit and repeatable, and the PAIS automatically manages the different manual and automatic activities involved. However, still many open issues remain. Therefore, we build a system comprising additional components as illustrated in the upper part of Fig. 1 .

The central component of our approach is a process configuration component that extends pre-defined base processes with pre-defined process fragments. To enable this in alignment with the context and meta data of the respective situation, we add a context mapping component as well as a comprehensive data model. Thus, it becomes possible to map various contextual factors into the SustainHub system and use them as parameters for process configuration to automatically generate process instances matching the properties of the respective situation.

However, to enable such a flexible automated approach, users must be able to pre-model many aspects involved in process configuration as illustrated in the lower part of Fig. 1. This comprises concepts for the context mapping: we apply context factors to capture contextual data. In turn, these can be mapped via context rules to a stable set of process parameters used as input for process configuration. The process configuration component employs bases processes and process fragments. The former are used as basis for a data collection process, while the latter are applied to extend that process situationally (see [2] for more details).

All entities based on these concepts have to be modeled correctly by users. To support this, we have introduced comprehensive correctness checks and put focus on keeping our approach as simple as possible. The first building block to achieve this is keeping the modeling of both base processes and process fragments directly in the PAIS. So the modeling is understandable and the processes can be checked by the PAIS automatically. In addition, users only have to model entities based on the three introduced context mapping concepts a small number of other entities for process configuration that govern where exactly to insert the fragments into the base processes. For both parts, we have also added correctness checks to ensure that only correct models come to execution. 


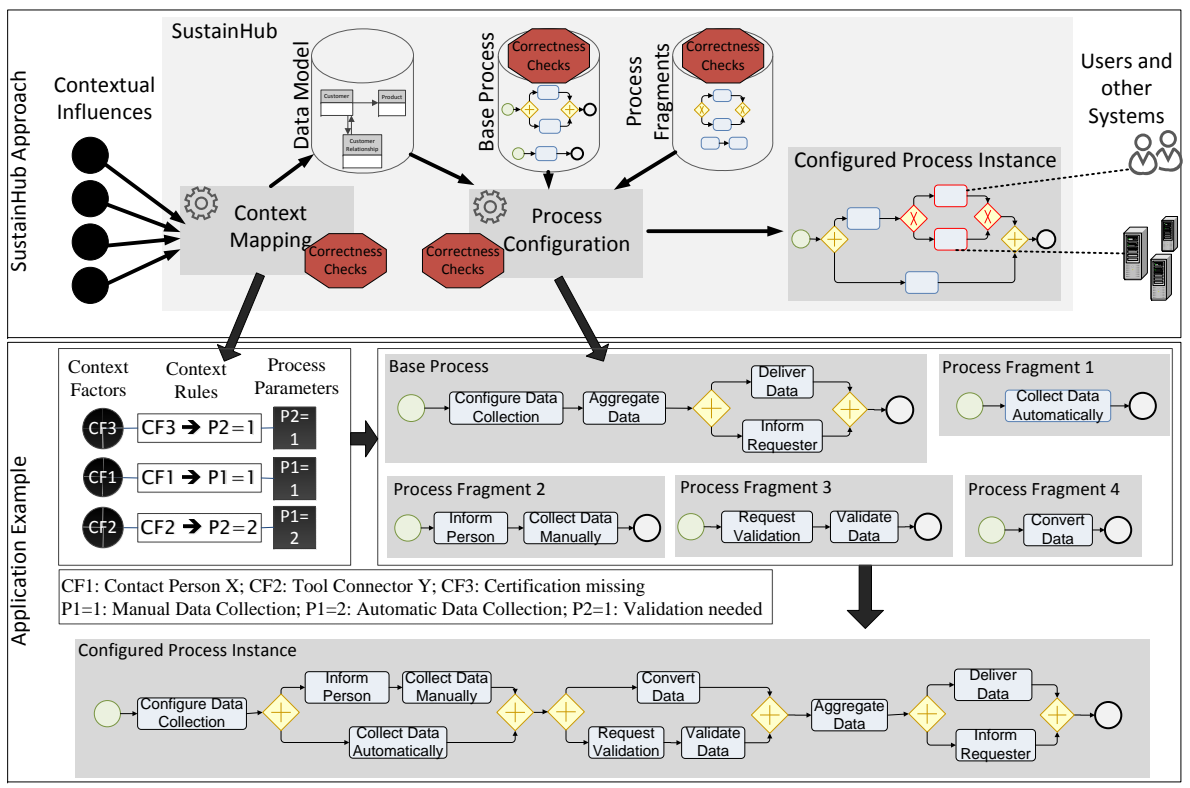

Fig. 1: Configurable Data Collection Approach in SustainHub

The lower part of Fig. 1 1 shows a simplified example of such a configuration use case from the industry: An automotive company wants to collect sustainability data relating to the quantity of lead contained in a specific product. This concerns two of the companies suppliers that deliver parts of that product. One is a bigger company with a dedicated system for managing sustainability data in place. The other is a smaller company with no system and no dedicated responsible for sustainability. For the smaller company, a service provider is needed that will validate the manually collected data ensuring its compliance with legal regulations. The system of the bigger company has its own data format that has to be explicitly converted in order to be usable. As depicted in Fig. 1. we apply three context factors to model that situation. These are mapped to values for two process parameters leading to the selection of four process fragments. Two of these are used for automatic and manual data collection while the two others enable external validation and data conversion. All fragments are integrated automatically into the base process to obtain a configured process instance. The latter first executes the data collection activities for both companies in parallel. After that, again in parallel, the data of one company is converted, while the data of the other company is validated externally.

\section{Related Work}

Various approaches for process variability exist. One aspect is the extension of process modeling languages to enable configurable process models [3]. Other ap- 
proaches like 4] target meta-modeling of various different aspects for process variability. Process fragments and their modeling, in particular, have been the focus of research, as in [5. Finally, the automated composition of executable processes from pre-defined process fragments has also been shown in approaches like $[6$. These approaches cover many areas of the process variability topic comprehensively. However, none of them has taken the context and users as our approach into account. The latter not only enables automated contextual process composition but also easy and error free modeling.

\section{Conclusion}

In this paper, we have sketched an approach for automated contextual process configuration. The latter not only enables the automatic processing of context data and the automatic composition of executable processes, it also puts focus on the users interacting with our system. Thus, we have created a lightweight way of modeling all necessary concepts and integrated a comprehensive set of correctness checks to support them. We have further applied this approach for complex data collection processes to supply chain communication in two domains. As part of future work we plan to extend our approach with runtime variability features and applying it in a comprehensive industrial evaluation.

\section{Acknowledgement}

The project SustainHub (Project No.283130) is sponsored by the EU in the 7th Framework Programme of the European Commission (Topic ENV.2011.3.1.9-1, Eco-innovation).

\section{References}

1. Grambow, G., Mundbrod, N., Steller, V., Reichert, M.: Challenges of Applying Adaptive Processes to Enable Variability in Sustainability Data Collection. In: SIMPDA 2013. (2013) 74-88

2. Grambow, G., Mundbrod, N., Steller, V., Reichert, M.: Towards Configurable Data Collection for Sustainable Supply Chain Communication. In: CAiSE'14 Forum. CEUR Workshop Proceedings, CEUR-WS.org (June 2014)

3. Ayora, C., Torres, V., Weber, B., Reichert, M., Pelechano, V.: Vivace: A Framework for the Systematic Evaluation of Variability Support in Process-Aware Information Systems. Information and Software Technology (May 2014)

4. Saidani, O., Nurcan, S.: Business Process Modeling: A Multi-perspective Approach Integrating Variability. In: BPMDS 2014. (2014) 169-183

5. Eberle, H., Leymann, F., Schleicher, D., Schumm, D., Unger, T.: Process Fragment Composition Operations. In: Proceedings of APSCC 2010, IEEE Xplore (December 2010) $1-7$

6. Murguzur, A., De Carlos, X., Trujillo, S., Sagardui, G.: Dynamic Composition of Pervasive Process Fragments. In: CAiSE 2014. (2014) 241-255 